\title{
A Virus’ Effect on Growth: HIV and African GDP Growth Rates
}

\author{
Brady Durst $^{\mathrm{a}}$, Filippo Rebessi ${ }^{\mathrm{a}}$
}

The sexually transmitted disease, HIV, is a vicious virus with no cure whose prevalence spans the entire globe, with daily diagnoses in every country. Those most affected with the terrors of the sickness lie in its birthplace of Africa, where one in ten carry the strain; and in some African countries more than a quarter of the population is HIV positive (Hacker, 2002). Compared to other world regions, Africa has a severe health crisis spawned from this relentless and incurable sickness. However, while the severity of the virus is widely known, its economic implications are not as apparent. It has been wondered, and seems intuitively correct, that a virus this deadly and prominent would have major implications on the level of output amongst highly infected countries (Dixon and McDonald, 2002). After all, a virus of this size would seem to affect numerous economic stimulating activities, such as the savings rate, labor force participation and worker determination. The expectation is to find HIV prevalence as having a negative and significant correlation to long run, per capita GDP growth rates in Africa; however, given the data, a statistical conclusion cannot be made to prove this occurrence. Instead, using linear regression, economic data infers HIV prevalence has little to no effect on Africa's sluggish GDP growth. The entirety of this paper expands on HIV prevalence and its relation to African GDP growth rates. A theoretical argument is given and followed by review of the data used to make the conclusion. Lastly, OLS Linear regression is used, seeking to find correlation between per capita GDP growth and HIV prevalence for 20 randomly selected African Countries. These two variables will be averaged over a span of 24 years, 1990-2013, to study effects on long run GDP growth.

\section{Theoretical arguments}

To better understand the reasoning behind HIV's expected effect on long run GDP growth, the Solow Growth Model, a model commonly used to measure economic output in developing countries, will be studied and manipulated. Specifically, the national savings and labor force variables will be analyzed, adjusted and entered into the model. The national savings variable will be adjusted due to its expected decrease in the presence of HIV. The claim being, a person afflicted with a life-threatening, incurable virus will change the way he or she financially plans for the future. A diagnosis of HIV would seem to lead to more rash decisions as the afflicted develop more of a "present-living" mindset. After all, with the thought of death in the near future, a sense to save for a financially sound future would likely be revoked and replaced with momentary decisions. Additionally, current savings would likely be spent on costly treatments rather than future, GDP stimulating investments.

To better illustrate this occurrence, a study conducted by the Reserve Bank of Australia found a major decrease in the savings rate of the elderly, specifically when comparing age cohorts 55-64 to those 65 and older (Beech). The study reveals the phenomenon of those closer to death saving a lower percentage of their income. These finding can be used analogously when predicting the savings habits of other neardeath cohorts. In an HIV afflicted community, where a significant sector of the population is death conscience, the aggregate saving habits would likely be affected.

A lower savings rate leads to lower long run economic growth and is best shown by manipulating the savings rate variable in the Solow Growth Model. The model consists of two equations: a production function and capital accumulation equation. The productivity equation is shown below:

(1) $Y=K^{\alpha} L^{1-\alpha}$

Where $\mathrm{Y}$ is the aggregate output, $\mathrm{K}$ represents physical capital and $\mathrm{L}$ is the labor force. This is the standard productivity equation used in the Solow Growth Model. The adjusted capital accumulation equation is shown below:
(1.1) $\dot{K}=s Y-\delta K ; s(\lambda)=s /(1+\lambda)$

Where $\dot{K}$ is the growth rate of physical capital, $s$ represents the new savings rate, $\mathrm{Y}$ is aggregate income, $\delta$ is the depreciation rate of capital, $\mathrm{K}$ is physical capital, $\lambda$ (a number between $0-1$ ) is the prevalence of HIV by percent population, and $\mathrm{s}$ is the unadjusted saving rate. The saving rate in the new capital accumulation equation has been manipulated to give a lower value depending on the HIV prevalence. The capital accumulation and productivity equations are then put into per capita terms and used to derive an equation representing the per capita output:

(1.2) $y=\left[\frac{s /(1+\lambda)}{\delta+n}\right] \frac{\alpha}{1-\alpha}$

Where $y$ is the per capita output, $\mathrm{s}$ is the savings rate, $\lambda$ is the prevalence of HIV by percent population, $\delta$ is the depreciation rate of physical capital and $n$ is the population growth rate. This equation highlights HIV's effect on output by decreasing the "would-be" savings rate, or the savings rate if HIV was not afflicting agents in the economy. As the equation suggests, the higher the $\lambda$ the lower the $\mathcal{y}$, long run GDP growth is decreased by the savings rate declining from an HIV prevalence in the economy.

The labor force variable is also affected from prevalence of HIV and in an analogous way to the savings rate. The higher the prevalence of HIV the less effectiveness of the labor force variable, thus lowering per capita GDP. The labor force variable is expected to be lower in countries with high HIV prevalence due to the toll taken on afflicted individuals. Those with HIV are likely to be less productive, feeling less motivation and incentive to excel in their occupation and, additionally, are not expected to remain in the workforce as long as those in other countries or may immediately leave the workforce upon diagnosis. Both factors lower the labor force and will be shown by manipulating the labor force variable, $L$, in the Solow Model, similar in fashion to the savings rate. 
Again, we start with two equations, the capital accumulation equation and the production function. Only the latter is adjusted from the original model and both are shown below:

(2) $\dot{K}=s Y-\delta K$

(2.1) $Y=K^{\alpha} \phi^{1-\alpha} ; \phi=(1-\lambda) L$

Where $\phi$, the new variable introduced into the model, represents the depreciation in the labor force variable, $\mathrm{L}$. Similar to above, $\lambda$ is HIV prevalence, $K$ is physical capital, $Y$ is the aggregate output and $\delta$ is the depreciation rate of capital. It is shown, the higher the HIV prevalence in this model, the lower the aggregate output, $Y$. The two above equations are put in per capita terms and used to derive a function representing per capita output:

$$
\text { (2.2) } y=(1-\lambda)\left[\frac{s}{\delta+n}\right] \frac{\alpha}{1-\alpha}
$$

The function predicts a lower per capita output as HIV prevalence increases. Again, it should be noted, the above equation is predicting those afflicted with HIV offer nothing to the workforce. Only a fraction of those afflicted would be less productive, but, for simplicity, we are using the most extreme case to better understand the effects of HIV on output.

Equation 2.2 and 1.2 are combined to summarize the effects of an HIV prevalence:

(3) $y=(1-\lambda)\left[\frac{s /(1+\lambda)}{\delta+n}\right] \frac{\alpha}{1-\alpha}$
Given the above function of per capita output, a country with non-zero HIV prevalence is hindered by a lower saving rate and a less productive labor force. A country afflicted with HIV is not producing to its maximum capacity by not allowing the labor force and savings rate reach their potential if HIV were a non-factor.

\section{Data Survey}

To analyze the effects of HIV prevalence on GDP, data was collected from the World Bank database for 20 randomly selected African countries. 20 countries were selected because the amount encompasses almost half of Africa's nations and is believed to be a fair and representative statistical sample. The selection process was conducted by assigning a number to each African country and subsequently using a random number generator to perform the 20 selections. Two variables were collected for the 20 African countries: HIV prevalence by percent population, ages 15-49, and per capita GDP growth rates. These two variables were collected from 1990-2013 and averaged for each country, giving one data point consisting of average HIV prevalence and its corresponding average per capita GDP for each country. The data set collected provides a wide range of HIV affected counties, including those severely afflicted with the virus and those with less than a prevalence of one percent. The Data is summarized in the following table:

Table 1. provides information about HIV average population percentage and the GDP average growth rate per country.

\begin{tabular}{|l|c|c|}
\hline \multicolumn{2}{|c|}{ HIV Prevalence and GDP growth rate for 20 African Countries } \\
\hline Country & $\begin{array}{c}\text { HIV Average by } \% \\
\text { Population }\end{array}$ & $\begin{array}{c}\text { GDP Average } \\
\text { Growth Rate }\end{array}$ \\
\hline Algeria & $0.10 \%$ & $0.97 \%$ \\
\hline Benin & $1.13 \%$ & $1.25 \%$ \\
\hline Cameroon & $4.20 \%$ & $-0.36 \%$ \\
\hline Central African Rep & $6.45 \%$ & $-0.90 \%$ \\
\hline Kenya & $7.37 \%$ & $0.42 \%$ \\
\hline Liberia & $2.08 \%$ & $2.79 \%$ \\
\hline Madagascar & $0.58 \%$ & $-0.78 \%$ \\
\hline Malawi & $14.80 \%$ & $1.63 \%$ \\
\hline Mali & $1.14 \%$ & $1.44 \%$ \\
\hline Morocco & $0.11 \%$ & $2.42 \%$ \\
\hline Mozambique & $7.45 \%$ & $3.64 \%$ \\
\hline Niger & $0.71 \%$ & $-0.30 \%$ \\
\hline Nigeria & $2.98 \%$ & $3.04 \%$ \\
\hline Senegal & $0.55 \%$ & $0.57 \%$ \\
\hline Sierra Leone & $0.95 \%$ & $1.82 \%$ \\
\hline Tanzania & $6.95 \%$ & $2.34 \%$ \\
\hline Togo & $2.83 \%$ & $0.03 \%$ \\
\hline Tunisia & $0.10 \%$ & $3.09 \%$ \\
\hline Zambia & $13.92 \%$ & $0.76 \%$ \\
\hline Zimbabwe & $21.02 \%$ & $-1.34 \%$ \\
\hline & & \\
\hline
\end{tabular}


Given the theoretical evidence, it is suggested that as HIV prevalence increases the GDP growth rate will correspondingly decrease. The countries with higher average HIV prevalence are suggested to show a lower average GDP growth rate. This phenomenon is simplified when looking at Malawi from the years 1995-2000. In this period of time as HIV prevalence increased, GDP rates dropped.

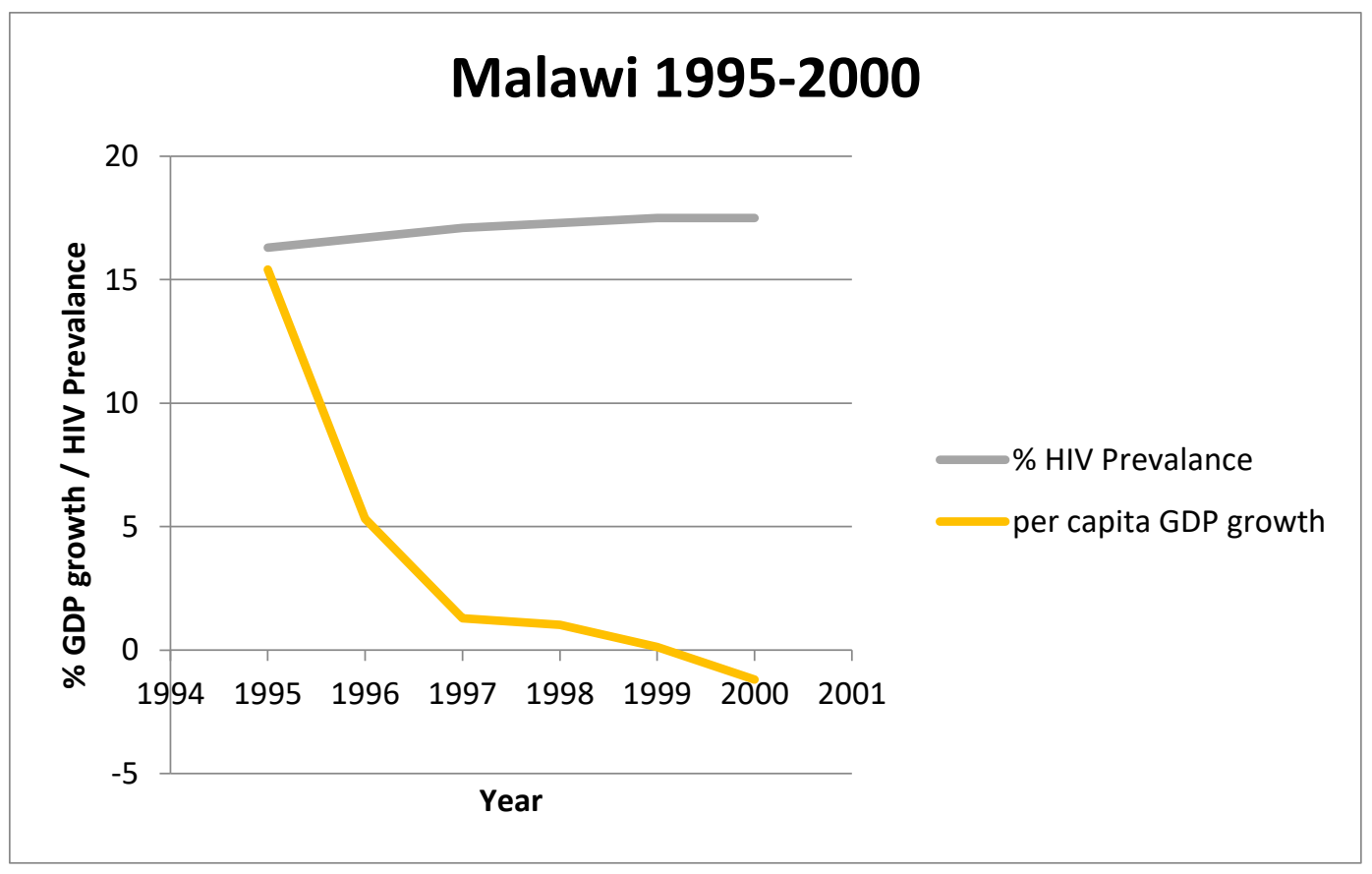

Figure 1 gives an extremely simplified version of the argument presented. In the example of Malawi, the correlation of HIV and GDP is consistent with the theoretical prediction; however, this data sample is extremely small and almost negligent when analyzing the entirety of Africa, especially in the context of long run growth rates. Therefore, in order to make a sound statistical conclusion, a regression will be conducted using the data given in table 1 .

\section{Empirical Analysis}

HIV's effect on GDP growth rates will be examined using the following regression equation for the data sample in question:

(4) $Y=a+\beta X+\varepsilon$

Where $\mathrm{Y}$ is the GDP per capita, $a$ is the intercept term, $X$ is the percent of population (aged 15-49) afflicted with HIV, $\mathcal{E}$ is the residual, and $\beta$ is the level of effect $X$ (HIV prevalence) has on Y (GDP per capita).

The regression for the coefficients of $\beta$ and $a$ will be conducted under the assumption of the null hypothesis being equal to zero $\left(H_{0}=0\right)$ and an alpha value equal to 0.05 $(\alpha=0.05)$. The $\mathrm{p}$-value of the intercept and beta term will be calculated under the null hypothesis assumption and compared to the alpha value.

Before collecting data and running the regression, the expectation for the beta term, further elaborated in the theoretical section, is to be negative and statistically significant ( $\mathrm{p}$-value $<\alpha=0.05$ ). In other words, increased HIV prevalence causes a decrease in the level of GDP per capita, as the two terms are negatively correlated. The residual, $\varepsilon$, is expected to be small, meaning the average distance each data point varies from the best-fit regression line will be low, making the model a good predictor of per capita GDP, given HIV prevalence.

The results of the regression are best shown summarized in the scatter plot below in figure 2: 


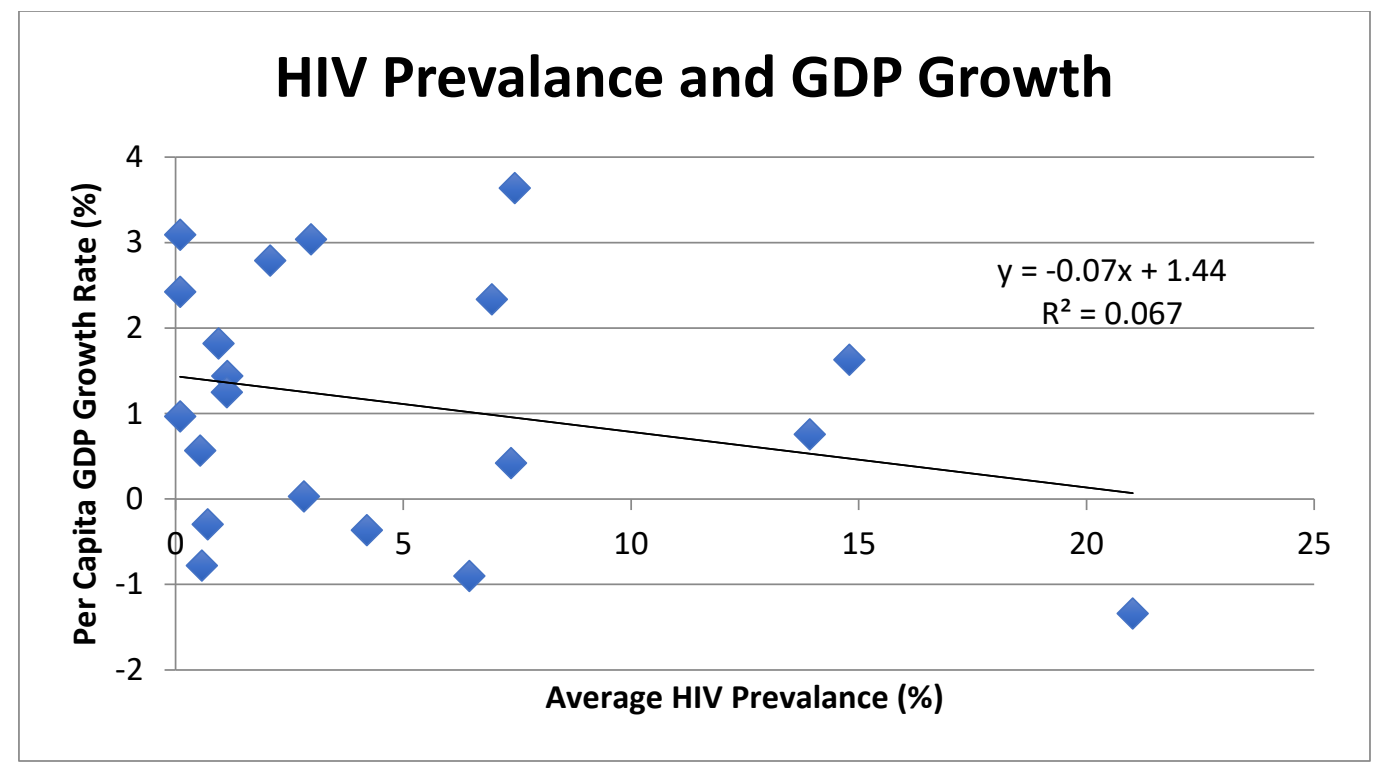

As shown in figure 2, the data points give an extremely scattered arrangement around the best-fit line. The graph is showing a high level of heterogeneity. Although the correlation between HIV prevalence and GDP is in the negative direction, the beta value is not statistically significant, and the scattered arrangement of the data points further labels this model as a bad predictor of long run, per capita GDP growth rates. The results of the regression are summarized below:

(4.1) $Y=1.44-0.07 X \pm 1.4 ; \beta$ 's p-value: 0.27

The regression shows HIV prevalence having a negative effect on GDP growth, however, as indicated by a p-value greater than the alpha level, the results are not statistically significant ( $\mathrm{p}$-value $<\alpha=0.05$ ). Given the current data set, no statistical conclusion can be made of the beta term being nonzero. Additionally, the residual for the regression is extremely high (1.4). This value is the average variation of the data points from the best-fit line and represents the model's ability to predict per capita GDP given HIV prevalence. This is also encompassed in the poor fit of the model. The coefficient of determination, $\mathrm{R}^{2}$, is close to zero, representing a model with poor prediction capabilities.

The regression was not in-line with the theoretical expectations. The beta value is close to zero and insignificant, while the value of the residual further labels the model as a failure by capturing its inability to make predictions. Some of the variation from theoretical prediction has been theorized to be from omitted-variable bias in the model, meaning an omitted variable in the error term may be affecting the dependent and independent variable; this claim is further elaborated in the Discussion and Conclusion section.

At this point, nothing can be said of HIV and GDP correlation, but it is worthwhile to test HIV's effect on the savings rate and labor force participation, since the two variables have been presented in the theoretical section to have a statistically significant effect on GDP. If it can be proven that HIV influences these two variables, it may lead to a conclusion of HIV consequentially affecting GDP, regardless of the above prediction.

More data was collected from the World Bank Database for savings rate by percent GDP and HIV prevalence. The savings rate is a variable calculating the percent of GDP invested by households in the economy. Data was collected for the same 20 countries analyzed in the previous regression and for the same timeframe. Again, results were averaged across the 23-year span. The results of the regression are shown in the following equation:

(4.2) $Y=12.5-0.37 X \pm 8.5 ; \beta$ 's p-value: 0.35

The regression gave a $\mathrm{p}$-value 0.35 to the beta term. The results are similar to the previous regression, HIV's effect is negative but labeled as insignificant and the amount of error, shown in the high residual, further labels the model as a futile predictor. The $\mathrm{p}$-value is even higher in this model, claiming HIV's effect on savings rate is less significant than its effect on GDP.

The final attempt to claim HIV has a statistically proven effect on GDP growth rates is to run a third regression, comparing HIV prevalence and labor force participation rate by population. The labor force participation data gives a percent of population involved in the labor force, retrieved from the World Bank Database and the same type of analysis in which values were averaged for the 23 -year period was used. It is reasonable to expect countries with a very high prevalence of HIV will have lower, on average, labor force participation. The results of the regression are summarized in the following equation:

\section{(4.3) $Y=60.4+0.64 X \pm 13.0 ; \beta$ 's p-value: 0.12}

The $\mathrm{p}$-value for the beta term, 0.12 , was again insignificant. However, although the p-value is insignificant, and the residual is high, there is a difference in this regression compared to the others: the beta term is positive. This term suggests as HIV prevalence increases, the labor force participation follows. This correlation is incoherent. It is incoherent to suggest a sickness causes a want to participate in labor. However, this data may provide reason to believe if HIV does diminish the labor force, it does only by a very small amount. For example, Zimbabwe, a country with a near quarter of the population afflicted with HIV, has the third highest labor participation rate of the countries analyzed. Although roughly $25 \%$ of the population is HIV positive, $80 \%$ of the population is involved in the labor force. Although unlikely, it is 
comprehensible to believe a chronic diagnosis, such as HIV, would lead some to reenter the workforce, since providing future compensation for loved ones could serve as motivation; however, this claim is left untested.

\section{Discussion and Conclusion}

It is expectation to find through statistical analysis that HIV prevalence will have a negative and significant effect on long run, per capita GDP growth rates in Africa; however, given the data, a statistical conclusion cannot be made. Although the theoretical idea of HIV's economic implications provides a framework for investigating the correlation, all three regressions conducted gave insignificant results, suggesting the effects of HIV on GDP as unproven.

It should be noted that endogenous bias is likely present in the regressions conducted. Specifically, simultaneity issues may be reason for misleading results. It is likely that HIV prevalence affects GDP growth, but also that GDP influences HIV prevalence. There is no direct causal relationship present in the variables. An example of this would be education on HIV prevention. Most likely the countries with higher GDP levels can better fund for education, indirectly lowering the level of HIV. This instance would mean higher levels of GDP would have lower HIV rates. Omitted variable bias may also lead to misguided results in the models, since it is probable an uncontrolled confounding variable is correlated with the independent variable, HIV prevalence and the dependent variable, GDP growth. No matter the situation, given the data set, there is no statistically significant correlation of HIV prevalence and per capita GDP growth rates.

While the data gave insignificant values, HIV may still have economic implications. The data available to run regressions on HIV inflicted countries is relatively small. Only ten African countries are considered to have a high prevalence of HIV. Ten data points cannot possibly encapsulate all of HIV's implications. Furthermore, GDP is a multifaceted variable that cannot be embodied by the study of one metric.
For example, HIV may be high in some African countries but their economic framework may be more sound than other African economies they are compared to. Another invalidity in the data used is the nature of the HIV variable itself. Without treatment, HIV remains dormant in the body for an average of ten years before serious health repercussions are present. The effects of the variable may not take its course until 10, 20 or even 30 years after one tests HIV positive. In the data analyzed HIV prevalence and corresponding growth rates were matched and averaged over a span of roughly 20 years, however, the HIV variable being gathered may be dormant in large sections of the economies included. In further research better results may be provided if past HIV rates are compared to GDP growth years afterward and economies of similar framework are used to better isolate the HIV variable.

Additionally, in future research more expansive regression analysis should be conducted to correct or partially control issues with endogenous bias, such as the two-stage least squares regression technique. Omitted variables that may have an effect, such as political corruption, should also be accounted for. A larger data sample should also be used to control for any outlier data present in the sample. Due to a limited number of countries with prevalence of HIV, it may be worthwhile to analyze data from a county or city level. Furthermore, because of the recent advances in HIV treatment, the overall consensus may be that the disease has little to no effect on GDP. It would be worthwhile to study other diseases' effects on GDP or previous timeframes when HIV diagnoses led to higher levels of mortality.

The results of the empirical analysis label the effect of HIV as insignificant. However, this may be subject to the nature of the data involved in the analysis and one cannot undermine the theoretical implications the deadliest virus in the world may have. In further research, data could be adjusted and controlled to isolate HIV's effects. Nonetheless, given the current data set and regressions studied, HIV cannot be considered to have an observable effect on long run, per capita GDP growth rates in African counties.

\section{References}

Beech, Amy. "The Distribution of Household Spending in Australia." The Reserve Bank of Australia. March 1, 2014. Accessed November 17, 2014.

Dixon, Simon, and Scott McDonald. "The Impact of HIV and AIDS on Africa's Economic Development." PMC, 2002. Accessed November 17, 2014. http://www.ncbi.nlm.nih.gov.

"Global Statistics." AIDS.gov. Accessed November 17, 2014.

"HIV/AIDS." World Health Organization. January 1, 2014. Accessed November 17, 2014.

Haacker, Markus. "The Economic Consequences of HIV/AIDS in Southern Africa." IMF, 2002. Accessed November $17,2014$. World Bank Database. January 1, 2014. Accessed November 14, 2014. http://data.worldbank.org/. 